\title{
Mathematical model of a part of an opened extra-high voltage electrical grid
}

\author{
Marek Lis ${ }^{1}$, Andriy Chaban ${ }^{2}$, Andrzej Szafraniec ${ }^{2,}{ }^{*}$, Radostaw Figura $^{2}$, Vitaliy Levoniuk ${ }^{3}$ \\ ${ }^{1}$ Czestochowa University of Technology, Faculty of Electrical Engineering, Poland \\ ${ }^{2}$ University of Technology and Humanities in Radom, Faculty of Transport and Electrical \\ Engineering, Poland \\ ${ }^{3}$ Lviv National Agricultural University, Ukraine
}

\begin{abstract}
In the paper, based on interdisciplinary approaches to modeling, a mathematical model of a part of an opened extra-high voltage electrical grid, which key elements are two long power transmission lines with distributed constants is presented. Within this framework the analysis of transient processes in power transmission lines in a single-line arrangement is carried out. The results of transient processes are displayed by means of figures; they are under ongoing research.
\end{abstract}

\section{Introduction}

The main objective of electrical grids is transmission and distribution of electricity, provided that its quality indicators are preserved. It is quite clear that the latter cannot be maintained without a reliable information on progress of transient processes in electrical grids. One of the most effective approaches to obtaining the above-mentioned information is use of mathematical modeling apparatus, which is based on the fundamental laws of applied physics; in our case it concerns electrodynamics. The key elements of extra-high voltage electrical grids are long power transmission lines with distributed constants, which leads to formation of boundary and mixed problems in mathematical terms. This, in turn, causes the problem of searching for boundary conditions for the telegrapher's equation. Therefore in the present paper we offer a technique of effective searching for boundary conditions for the wave equation, which, as a result, makes it possible to study transient wave processes in electrical grids.

\section{Analysis of recent research}

There are many scientific papers devoted to analysis of transient processes in power systems. Let us consider some of them which are related to the current paper.

In the article [1], the questions of mathematical models and macro-models of power transmission lines development are discussed. A discrete macro-model of a single-phase

* Corresponding author: a.szafraniec@uthrad.pl 
power transmission line with the use of "black box" is developed. The model description, the order of its development and research findings are presented.

In [2], mathematical models of two - and three-wire alternating-current transmission lines are developed, with the help of which different nature of transient processes is analysed using the software complex ATP-EMTP.

It is obvious that not all these approaches to development of mathematical models of transient processes in long power transmission lines enable to take into account influence of other elements of a power system on processes in a line. Yet models including this feature are implemented in ATP-EMTP and MATLAB/Simulink software complexes, which does not always provide for more adequate result, especially when electromagnetic models of $\Psi$ - A- type elements are used as models of power systems elements, and especially when the circular and field mathematical models of the latter are used [3, 4].

The aim of the paper is analysis of transient processes in the part of opened extra-high voltage electrical grid whose key elements are two long power transmission lines with distributed constants power systems.

\section{System description}

The present paper describes a model of an opened extra-high voltage electrical grid (Fig. 1). Power transmission lines are presented in the form of equivalent circuits with distributed constants. They combine power systems for parallel operation, which are presented by their own electromotive force, internal resistance and inductance.

We study only symmetrical modes and this allows us to consider a three-phase symmetrical electrical grid in a single-line arrangement.

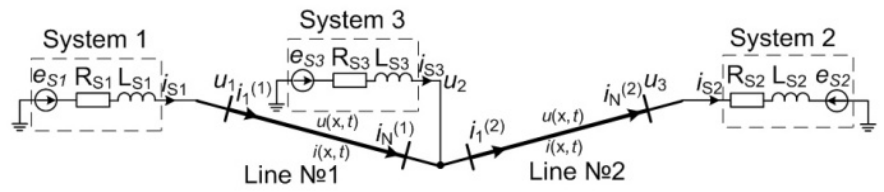

Fig. 1. A calculated equivalent circuit of the studied part of an opened electrical grid.

For the purposes of analysing transient processes in the part of the opened electrical grid, shown in Fig. 1, we suggest using a modified Hamilton-Ostrogradsky principle [5]

The extended functionality of the mathematical operation for the studied system according to Hamilton-Ostrogradskii will be similar to that presented in [5-8]:

$$
S=\int_{0}^{t_{1}} L^{*} d t+\int_{0}^{t_{1}} \int_{l} L_{l} d l d t, I=\int_{l} L_{l} d l
$$

where $S$ - action according to Hamilton-Ostrogradskii, $L^{*}$ - extended Lagrange function, $L_{1}$ - linear density of the modified Lagrange function, $I$ - energy functional.

The extended Lagrange function is presented in [5].

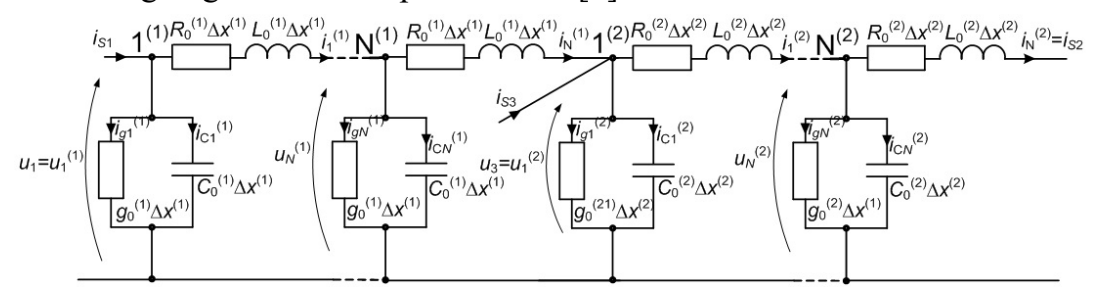

Fig. 2. The equivalent circuit of power transmission lines connection with distributed constants. 


$$
L^{*}=\tilde{T}^{*}-P^{*}+\Phi^{*}-D^{*}
$$

where $L^{*}$ - extended Lagrange function, $\widetilde{T}^{*}$ - kinetic coenergy, $P^{*}-$ potential energy, $\Phi^{*}$ - energy dissipation, $D^{*}$ - energy of outside nonpotential forces [9].

Let us write down elements of the extended Lagrange function [5]

$$
\begin{gathered}
T^{*}=\frac{L_{S 1} i_{S 1}^{2}}{2}+\frac{L_{S 1} i_{S 2}^{2}}{2}+\frac{L_{S 3} i_{S 3}^{2}}{2} \\
\Phi^{*}=\frac{1}{2} \int_{0}^{t}\left(R_{S 1} i_{S 1}^{2}+R_{S 2} i_{S 2}^{2}+R_{S 3} i_{S 3}^{2}\right) d \tau \\
D^{*}=\int_{0}^{t}\left(e_{S 1} i_{S 1}+e_{S 2} i_{S 2}+e_{S 3} i_{S 3}\right) d \tau \\
\frac{\partial T^{\langle k\rangle}}{\partial x} \equiv T_{l}^{\langle k\rangle}=\frac{L_{0}^{\langle k\rangle} Q_{t}^{2\langle k\rangle}(x, t)}{2}, \frac{\partial P^{\langle k\rangle}}{\partial x} \equiv P_{l}^{\langle k\rangle}=\frac{1}{2 C_{0}^{\langle k\rangle}} Q_{x}^{2\langle k\rangle}(x, t)
\end{gathered}
$$

where

$$
\begin{gathered}
Q_{t}^{\langle k\rangle} \equiv \frac{\partial Q^{\langle k\rangle}(x, t)}{\partial t}=i^{\langle k\rangle}(x, t) ; \quad Q_{x}^{\langle k\rangle} \equiv \frac{\partial Q^{\langle k\rangle}(x, t)}{\partial x} \\
\frac{\partial \Phi^{\langle k\rangle}}{\partial x} \equiv \Phi_{l}^{\langle k\rangle}=\Phi_{l 3}^{\langle k\rangle}-\Phi_{l B}^{\langle k\rangle}=\int_{0}^{t}\left(\frac{R_{0}^{\langle k\rangle}}{2} Q_{t}^{2\langle k\rangle}(x, t)-\frac{g_{0}^{\langle k\rangle}}{2 C_{0}^{2\langle k\rangle}} Q_{x}^{2\langle k\rangle}(x, t)\right)_{\mid t=\tau} d \tau ; \quad k=1,2
\end{gathered}
$$

where $L_{S 1}, L_{S 2}, L_{S 3}$ - inductance of the systems 1,2 and 3, respectively; $R_{S 1}, R_{S 2}, R_{S 3}$ - active resistance of the systems 1,2 and 3 , respectively; $e_{S 1}, e_{S 2}, e_{S 3}$ - electromotive force of the systems 1,2 and $3 ; i_{S 1}, i_{S 2}, i_{S 3}$ - current of the systems 1,2 and 3 , respectively; $i(x, t)$ current in the line; $R_{0}, g_{0}, C_{0}, L_{0}$ - distributed constants of the line; $\Phi_{l 3}$ - linear density of external energy dissipation; $\Phi_{l B}$ - linear density of internal energy dissipation; $Q(x, t)$ - the line charge; $k$ - the line number.

One can acquaint oneself with the method of the Similar equations are presented in [3, $10,11]$, for example. Therefore, to articlee synthetic, we suggest finished equations of the electromagnetic state of the object, Fig. 1.

$$
\begin{aligned}
\frac{\partial v^{\langle k\rangle}}{\partial t}=\left(C_{0}^{\langle k\rangle} L_{0}^{\langle k\rangle}\right)^{-1}\left(\frac{\partial^{2} u\langle k\rangle}{\partial x^{2}}-\right. & \left.\left(g_{0}^{\langle k\rangle} L_{0}^{\langle k\rangle}+C_{0}^{\langle k\rangle} R_{0}^{\langle k\rangle}\right) v^{\langle k\rangle}-g_{0}^{\langle k\rangle} R_{0}^{\langle k\rangle} u^{\langle k\rangle}\right), \frac{\partial u^{\langle k\rangle}}{\partial t}=v^{\langle k\rangle} \\
\frac{d i_{S 1}}{d t} & =\frac{1}{L_{S 1}}\left(e_{S 1}-R_{S 1} i_{S 1}-u_{1}\right) \\
\frac{d i_{S 2}}{d t} & =\frac{1}{L_{S 2}}\left(u_{2}-R_{S 2} i_{S 2}-e_{S 2}\right) \\
\frac{d i_{S 3}}{d t} & =\frac{1}{L_{S 3}}\left(e_{S 3}-R_{S 3} i_{S 3}-u_{3}\right)
\end{aligned}
$$


where $u_{1}, u_{2}, u_{3}$ - substation busbars voltage of the power systems 1, 2 and 3 respectively.

Kirchhoff's Second Law for electrical circuits with distributed constants is used as boundary conditions for (9):

$$
-\frac{\partial u^{\langle k\rangle}}{\partial x}=R_{0}^{\langle k\rangle} i^{\langle k\rangle}+L_{0}^{\langle k\rangle} \frac{\partial i^{\langle k\rangle}}{\partial t}
$$

We will write down (9) and (13) in discrete space for the j-node of the line (using the concept of the central derivative):

$$
\begin{gathered}
\frac{d v_{j}^{\langle k\rangle}}{d t}=\left(C_{0}^{\langle k\rangle} L_{0}^{\langle k\rangle}\right)^{-1}\left(\frac{u_{j-1}^{\langle k\rangle}-2 u_{j}^{\langle k\rangle}+u_{j+1}^{\langle k\rangle}}{(\Delta x\langle k\rangle)^{2}}-\left(g_{0}^{\langle k\rangle} L_{0}^{\langle k\rangle}+C_{0}^{\langle k\rangle} R_{0}^{\langle k\rangle}\right) v_{j}^{\langle k\rangle}-g_{0}^{\langle k\rangle} R_{0}^{\langle k\rangle} u_{j}^{\langle k\rangle}\right) \\
u_{1}^{\langle k\rangle}=\left.u(x, t)\right|_{x=0} ; u_{N}^{\langle k\rangle}=\left.u(x, t)\right|_{x=l} ; u_{1}^{\langle 1\rangle} \equiv u_{1} ; u_{1}^{\langle 2\rangle} \equiv u_{3} ; u_{N}^{\langle 1\rangle} \neq u_{3} ; u_{N}^{\langle 2\rangle} \neq u_{2} \\
-\frac{u_{j+1}^{\langle k\rangle}-u_{j-1}^{\langle k\rangle}}{2 \Delta x^{\langle k\rangle}}=R_{0}^{\langle k\rangle} i_{j}^{\langle k\rangle}+L_{0}^{\langle k\rangle} \frac{d i_{j}^{k k\rangle}}{d t} \\
\frac{d u_{j}^{\langle k\rangle}}{d t}=v_{j}^{\langle k\rangle}, j=1, \ldots, N ; \quad k=1,2
\end{gathered}
$$

Having analyzed the equation (14), it can be seen that in order to find the voltage on the first and the last nodes of discretization, it is necessary to find the unknown voltages in the fictitious nodes $u_{0}^{\langle k\rangle}$ and $u_{N+1}^{\langle k\rangle}$. Let's search for the voltage.

Let's note the equation of scleronomic constraint (see Fig. 2):

$$
\begin{gathered}
i_{S 1}-i_{1}^{\langle 1\rangle}-i_{g 1}^{\langle 1\rangle}-i_{C 1}^{\langle 1\rangle}=0 ; \quad i_{g 1}^{\langle 1\rangle}=\Delta x^{\langle 1\rangle} g_{0}^{\langle 1\rangle} u_{1}^{\langle 1\rangle} \\
i_{C 1}^{\langle 1\rangle}=\Delta x^{\langle 1\rangle} C_{0}^{\langle 1\rangle} \frac{d u_{1}^{\langle 1\rangle}}{d t}=\Delta x^{\langle 1\rangle} C_{0}^{\langle 1\rangle} v_{1}^{\langle 1\rangle}
\end{gathered}
$$

The next step is to differentiate (17) and (18) with respect to time:

$$
\begin{gathered}
\frac{d i_{S 1}}{d t}-\frac{d i_{1}^{\langle 1\rangle}}{d t}-\frac{d i_{g 1}^{\langle 1\rangle}}{d t}-\frac{d i_{C 1}^{\langle 1\rangle}}{d t}=0 ; \quad \frac{d i_{g 1}^{\langle 1\rangle}}{d t}=\Delta x^{\langle 1\rangle} g_{0}^{\langle 1\rangle} v_{1}^{\langle 1\rangle} \\
\frac{d i_{C 1}^{\langle 1\rangle}}{d t}=\Delta x^{\langle 1\rangle} C_{0}^{\langle 1\rangle} \frac{d v_{1}^{\langle 1\rangle}}{d t}
\end{gathered}
$$

Now, based on (15) and Fig. 2, we will write:

$$
\frac{d i_{1}^{\langle 1\rangle}}{d t}=\frac{1}{L_{0}^{\langle 1\rangle}}\left(\frac{u_{0}^{\langle 1\rangle}-u_{2}^{\langle 1\rangle}}{2 \Delta x^{\langle 1\rangle}}-R_{0}^{\langle 1\rangle} i_{1}\langle 1\rangle\right)
$$




$$
\begin{aligned}
& \frac{d i_{N}^{\langle 1\rangle}}{d t}=\frac{1}{L_{0}^{\langle 1\rangle}}\left(\frac{u_{N-1}^{\langle 1\rangle}-u_{N+1}^{\langle 1\rangle}}{2 \Delta x^{\langle 1\rangle}}-R_{0}^{\langle 1\rangle} i_{N}^{\langle 1\rangle}\right) \\
& \frac{d i_{N}^{\langle 1\rangle}}{d t}=\frac{1}{L_{0}^{\langle 1\rangle} \Delta x^{\langle 1\rangle}}\left(u_{N}^{\langle 1\rangle}-R_{0}^{\langle 1\rangle} \Delta x^{\langle 1\rangle} i_{N}^{\langle 1\rangle}-u_{1}^{\langle 2\rangle}\right)
\end{aligned}
$$

Substituting (10) from (19) to the first equation, and equations (20) and (21) from (19) to the second equation, we will obtain:

$$
\frac{1}{L_{S 1}}\left(e_{S 1}-R_{S 1} i_{S 1}-u_{1}\right)-\frac{1}{L_{0}^{\langle 1\rangle}}\left(\frac{u_{0}^{\langle 1\rangle}-u_{2}^{\langle 1\rangle}}{2 \Delta x^{\langle 1\rangle}}-R_{0}^{\langle 1\rangle} i_{1}{ }^{11\rangle}\right)-\Delta x^{\langle 1\rangle} g_{0}^{\langle 1\rangle} v_{1}^{\langle 1\rangle}-\Delta x^{\langle 1\rangle} C_{0}^{\langle 1\rangle} \frac{d v_{1}^{\langle 1\rangle}}{d t}=0
$$

Substituting (14) to (24), which is written for the first discretization node for the first line and deriving the voltage from there, we will obtain:

$$
\begin{aligned}
u_{0}^{\langle 1\rangle}=\frac{2 \Delta x^{\langle 1\rangle} L_{0}^{\langle 1\rangle}}{3}\left[\frac{1}{L_{S 1}}\left(e_{S 1}-R_{S 1} i_{S 1}-u_{1}^{\langle 1\rangle}\right)+\left(\frac{\Delta x^{\langle 1\rangle} g_{0}^{\langle 1\rangle} R_{0}^{\langle 1\rangle}}{L_{0}^{\langle 1\rangle}}+\frac{2}{L_{0}^{\langle 1\rangle} \Delta x^{\langle 1\rangle}}\right) u_{1}^{\langle 1\rangle}-\frac{1}{2 L_{0}^{\langle 1\rangle} \Delta x^{\langle 1\rangle}} u_{2}^{\langle 1\rangle}-\right. \\
\\
\left.-\left(\Delta x^{\langle 1\rangle} g_{0}^{\langle 1\rangle}-\frac{\Delta x^{\langle 1\rangle}\left(g_{0}^{\langle 1\rangle} L_{0}^{\langle 1\rangle}+C_{0}^{\langle 1\rangle} R_{0}^{\langle 1\rangle}\right)}{L_{0}^{\langle 1\rangle}}\right) v_{1}^{\langle 1\rangle}+\frac{R_{0}^{\langle 1\rangle}}{L_{0}^{\langle 1\rangle}} i_{1}^{\langle 1\rangle}\right]
\end{aligned}
$$

Equations (22) and (23) against each other will produce:

$$
\frac{1}{L_{0}^{\langle 1\rangle}}\left(\frac{u_{N-1}^{\langle 1\rangle}-u_{N+1}^{\langle 1\rangle}}{2 \Delta x^{\langle 1\rangle}}-R_{0}^{\langle 1\rangle} i_{N}^{\langle 1\rangle}\right)=\frac{\left(u_{N}^{\langle 1\rangle}-R_{0}^{\langle 1\rangle} \Delta x^{\langle 1\rangle} i_{N}^{\langle 1\rangle}-u_{1}^{\langle 2\rangle}\right)}{L_{0}^{\langle 1\rangle} \Delta x^{\langle 1\rangle}}
$$

Voltage in the fictitious node $u_{N+1}^{\langle 1\rangle}$ results:

$$
u_{N+1}^{\langle 1\rangle}=-2\left(u_{N}^{\langle 1\rangle}-u_{1}^{\langle 2\rangle}\right)+u_{N-1}^{\langle 1\rangle}
$$

Due to space constraints, we will not provide findings for voltage in fictitious nodes of the second line, but will provide the finite expressions:

$$
\begin{gathered}
u_{0}^{\langle 2\rangle}=\frac{2 \Delta x^{\langle 2\rangle} L_{0}^{\langle 2\rangle}}{3}\left[\frac{1}{L_{S 3}}\left(e_{S 3}-R_{S 3} i_{S 3}-u_{1}^{\langle 2\rangle}\right)+\left(\frac{\Delta x^{\langle 2\rangle} g_{0}^{\langle 2\rangle} R_{0}^{\langle 2\rangle}}{L_{0}^{\langle 2\rangle}}-\frac{1}{L_{0}^{\langle 1\rangle} \Delta x^{\langle 1\rangle}}+\frac{2}{L_{0}^{\langle 2\rangle} \Delta x^{\langle 2\rangle}}\right) u_{1}^{\langle 2\rangle}-\right. \\
\left.-\frac{1}{2 L_{0}^{\langle 2\rangle} \Delta x} x^{\langle 2\rangle} u_{2}^{\langle 2\rangle}-\left(\Delta x^{\langle 2\rangle} g_{0}^{\langle 2\rangle}-\frac{\Delta x^{\langle 2\rangle}\left(g_{0}^{\langle 2\rangle} L_{0}^{\langle 2\rangle}+C_{0}^{\langle 2\rangle} R_{0}^{\langle 2\rangle}\right)}{L_{0}^{\langle 2\rangle}}\right) v_{1}^{\langle 2\rangle}+\frac{1}{L_{0}^{\langle 1\rangle} \Delta x^{\langle 1\rangle}} u_{N}^{\langle 1\rangle}+\frac{R_{0}^{\langle 2\rangle}}{L_{0}^{\langle 2\rangle}} i_{1}^{\langle 2\rangle}-\frac{R_{0}^{\langle 1\rangle}}{L_{0}^{\langle 1\rangle}} i_{N}^{\langle 1\rangle}\right] \\
u_{N+1}^{\langle 2\rangle}=-2 L_{0}^{\langle 2\rangle} \Delta x^{\langle 2\rangle}\left[\frac { 1 } { L _ { S 2 } + L _ { 0 } ^ { \langle 2 \rangle } \Delta x ^ { \langle 2 \rangle } } \left(u_{N}^{\langle 2\rangle}-i_{N}^{\langle 2\rangle}\left(R_{0}^{\langle 2\rangle} \Delta x^{\langle 2\rangle}+\right.\right.\right.
\end{gathered}
$$




$$
\left.\left.\left.+R_{S 2}\right)-e_{S 2}\right)-\frac{1}{2 L_{0}^{\langle 2\rangle} \Delta x^{\langle 2\rangle}} u_{N-1}^{\langle 2\rangle}+\frac{R_{0}^{\langle 2\rangle}}{L_{0}^{\langle 2\rangle}} i_{N}^{\langle 2\rangle}\right]
$$

The current in the line can be found by discretizing (13) by the finite difference method, using the concept of the right derivative [5]:

$$
\frac{d i_{j}^{\langle k\rangle}}{d t}=\frac{1}{L_{0}^{\langle k\rangle} \Delta x}\left(u_{j}^{\langle k\rangle}-u_{j+1}^{\langle k\rangle}\right)-\frac{R_{0}^{\langle k\rangle}}{L_{0}^{\langle k\rangle}} i_{j}^{\langle k\rangle}, j=1, \ldots, N ; \quad k=1,2
$$

The following system of differential equations is subject to joint integration: (10) - (12), (14), (16), (30) including (25), (27) - (29).

\section{Computer simulation findings}

A computer simulation was conducted to study transient processes during system switching in a steady-state mode $[10,12,13]$.

The parameters of the equivalent circuit elements in Fig. 1 correspond to the parameters of the real part of $750 \mathrm{kV}$ electrical grid. The line No. 1 is $282 \mathrm{~km}$ long, and the line No. 2 is $476 \mathrm{~km}$ long. The parameters of the grid elements are as follows.

The parameters of two long lines with distributed constants are as follows: $e_{S 1}=$ $622 \sin \left(\omega t+10.3^{\circ}\right) \mathrm{kV}, e_{S 2}=581 \sin (\omega t) \mathrm{kV}, e_{S 3}=600 \sin \left(\omega t+4.9^{\circ}\right) \mathrm{kV}, R_{S 1}=2.3 \mathrm{Om}$, $R_{S 2}=2.5 \mathrm{Om}, R_{S 3}=2.05 \mathrm{Om}, L_{S 1}=0.15 \mathrm{H}, L_{S 2}=0.145 \mathrm{H}, L_{S 3}=0.165 \mathrm{H}$. The parameters of two long lines with distributed constants are as follows: $R_{0}=1.9 \cdot 10^{-5} \mathrm{Om} / \mathrm{m}, L_{0}=$ $9.24 \cdot 10^{-7} \mathrm{H} / \mathrm{m}, \mathrm{C}_{0}=1.3166 \cdot 10^{-11} \mathrm{~F} / \mathrm{m}, g_{0}=3.25 \cdot 10^{-11} \mathrm{Sm} / \mathrm{m}$.

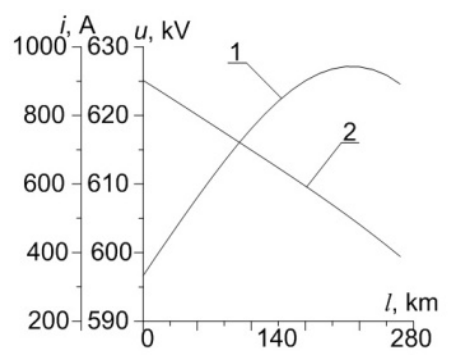

Fig. 3. The spatial distribution of voltage (1) and current (2) in the line No. 1 at the time $t=0.003 \mathrm{~s}$.

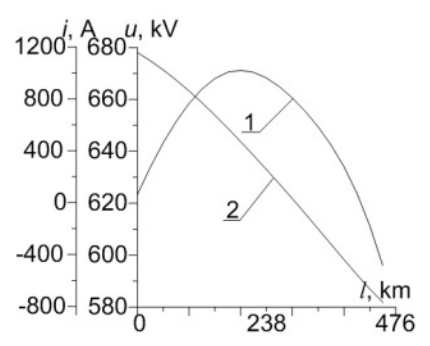

Fig. 4. The spatial distribution of voltage (1) and current (2) in the line No. 2 at the time $t=0.003 \mathrm{~s}$.

Figures 3 and 4 show the spatial distribution of voltage and current functions in the lines No. 1 and No. 2 at the time $t=0.003 \mathrm{~s}$, respectively. It can be observed that the distributions in the lines are to some extent similar. Differences in the distributions can be explained by the different lengths of these lines and parameters of the systems which they connect. 


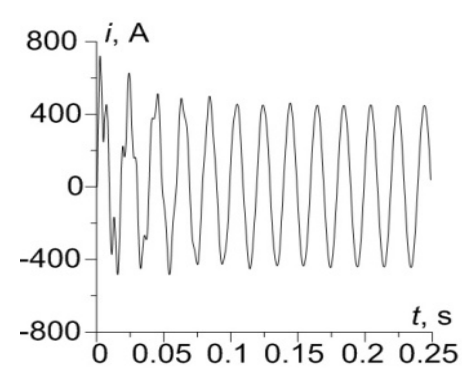

Fig. 5. Current at the midpoint of the line No. 1.

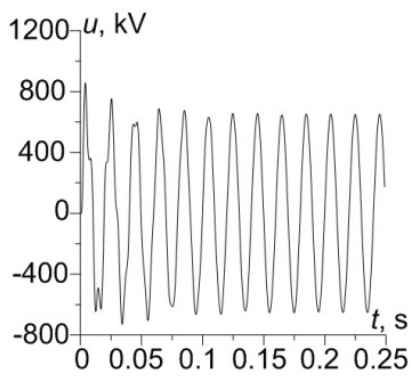

Fig. 6. Voltage at the midpoint of the line No. 2.

Fig. 5 and 6 show the current transient process at the midpoint of the line No. 1 and voltage at the midpoint of the line No. 2, respectively. It can be seen that the transient process is almost completed in $0.15 \mathrm{~s}$ after the system start-up.

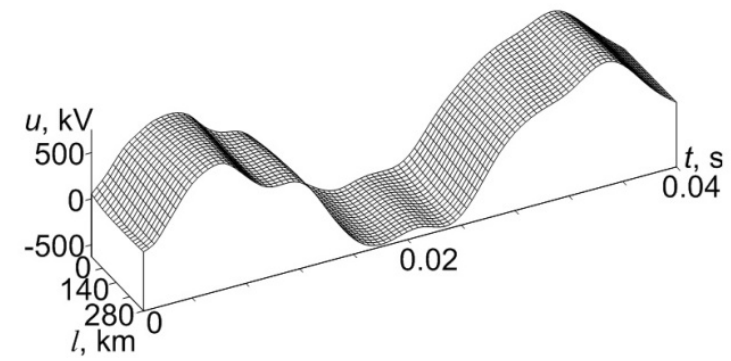

Fig. 7. Temporal-spatial distribution of voltage functions in the line No. 1 at the time $t[0 ; 0.04] \mathrm{s}$.

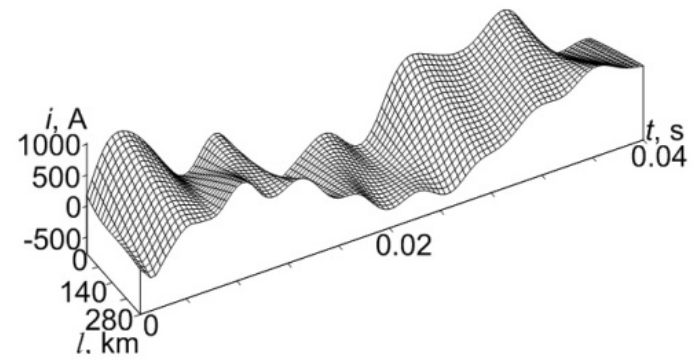

Fig. 8. Temporal-spatial distribution of voltage functions in the line No. 1 at the time $t[0 ; 0.04] \mathrm{s}$.

Fig. 7 and 8 show voltage and current of the line No. 1 as a function of temporal-spatial coordinates. These figures are presented in $3 \mathrm{D}$ format. The high informative value of the latter should be noted; it lies in the fact that both spatial and temporal coordinates form a three-dimensional space.

\section{Conclusion}

1. Application of a modified integral variational principle of Hamilton-Ostrogradskiy allows to construct mathematical models of quite complex dynamical systems; in our particular case, a mathematical model of a part of the opened extra-high voltage electrical grid was constructed which consists of two long power transmission lines with distributed constants which combine power systems for parallel operation. 
2. The mathematical model of the long power transmission line with distributed constants allows to analyze complex transient processes in such lines, which are almost impossible to take into account on the basis of a circular equivalent circuit of a long line scheme, because the partial differential equations help to describe physical processes taking into account environment continuity, in particular, to calculate propagation velocity of electromagnetic waves in long lines, to consider different wave processes, et caetera.

\section{References}

1. P. Stakhiv, Y. Kozak, O. Hoholyuk, Discrete mathematical macromodel of electric transmission line, Przegląd elektrotechniczny, 4, 272-274, (2013)

2. A. Nayir, Simulation of transient processes on overvoltage in electric transmission lines using ATP-EMTP, Turkish Journal of Electrical Engineering \& Computer Sciences, 5, 1553-1556, (2013)

3. A. Czaban, M. Lis, M. Chrzan, A. Szafraniec, V. Levoniuk, Mathematical modelling of transient processes in power supply grid with distributed parameters, Przegląd elektrotechniczny, 94, $17-20,(2018)$

4. R. Ortega, A. Loria, P.J. Nicklasson, H. Sira-Ramirez, Passivity-Beast Control of Euler-Lagrange Systems: Mechanical, Electrical and Electromechanical Applications. (Springer Verlag, London 1998)

5. A. Chaban, Principle Gamiltona-Ostrogradskogo in elektromehanichnih systems, (Soroki, Lviv 2015)

6. A. Szafraniec, Modelowanie matematyczne procesów oscylacyjnych w napędzie elektrohydraulicznym o podatnej transmisji ruchu, Przegląd Elektrotechniczny, 12, 167-171, (2017)

7. P. Pukach, V. Ilkiv, Z. Nytrebych, M. Vovk, On nonexistence of global in time solution for a mixed problem for a nonlinear evolution equation with memory generalizing the Voigt - Kelvin rheological model, Opuscula Mathematica, 5, 735-753, (2017)

8. Z. Łukasik, A. Czaban, A. Szafraniec, V. Żuk, The mathematical model of the drive system with asynchronous motor and vertical pump, Przegląd Elektrotechniczny, 1, 133-138, (2018)

9. Z. Łukasik, A. Czaban, A. Szafraniec, Mathematical model of asynchronous pump drive with distributed mechanical parameters, Przegląd Elektrotechniczny, 6, 155-159, (2018)

10. A. Czaban, M. Lis, J. Sosnowski, W. Lewoniuk, Mathematical model of the double conductor power line using a modified Hamilton's principle, Electric machines, 109, $31-36,(2016)$

11. A. Szafraniec, Mathematical Model of Asynchronous Pump Drive and Power Transformer Drive System with Complex Motion Transmission, Control of Power Systems 13th International Scientific Conference CPS, Tatranské Matliare - Tatranská Lomnica, (2018)

12. A. Czaban, A. Szafraniec, M. Lis, V. Levoniuk, H. Lysiak, R. Figura, Transient Processes Analysis in a Part of an Power Grid during a Automatic Reclosing Cycle, Control of Power Systems 13th International Scientific Conference CPS, Tatranské Matliare - Tatranská Lomnica, (2018) 
13. P. Sowa, R. Kumala, K. Łuszcz, Modeling of Power System Components During Electromagnetic Transients, International Journal of Innovative Science, Engineering \& Technology, 10, 716-719, (2014) 